\title{
The interactive effects of stress and coping style on cognitive function in patients with schizophrenia
}

This article was published in the following Dove Medical Press journal: Neuropsychiatric Disease and Treatment

\author{
Xiaodan Zhu' \\ Xuebing $\mathrm{Xu}^{2}$ \\ Chao $\mathrm{Xu}^{2}$ \\ Jingyi Zhang ${ }^{2}$ \\ Xiaofeng Zhang ${ }^{2}$ \\ $\mathrm{Li} \mathrm{Ma}{ }^{2}$ \\ Juan $\mathrm{Liu}^{3}$ \\ Kefang Wang' \\ 'Division of Nursing Fundamentals, \\ School of Nursing, Shandong \\ University, Jinan, Shandong, China; \\ ${ }^{2}$ Inpatient Department, Ningxia \\ Mental Health Center, Ningxia \\ Ning-An Hospital, Yinchuan, Ningxia, \\ China; ${ }^{3}$ Division of Humanities, \\ School of Nursing, Ningxia Medical \\ University, Yinchuan, Ningxia, China
}

Correspondence: Kefang Wang School of Nursing, Shandong University, Wenhua Xi Road 44, Jinan, Shandong 2500 I2, China

Tel $+8653 \mid 8838220$ I

Fax +86 53I 82942003

Email wangkf@email.sdu.edu.cn

Juan Liu

School of Nursing, Ningxia Medical

University, Shengli Road II60, Yinchuan,

Ningxia 750004, China

Tel/fax +869516980191

Email ryuken05I8@I63.com
Purpose: Previous studies have not determined the interactive effects of stress and coping style on cognitive function in patients with schizophrenia, and the current studies have been restricted to the relationship between stress and stress response, which may be associated with cognitive impairment in individuals with schizophrenia. The present research was aimed to determine whether stress is related to cognitive function in patients with schizophrenia. In addition, this research further investigates the moderating effects of coping style on the relationship between stress and cognitive function in patients with schizophrenia on the basis of stress and coping theory.

Patients and methods: Our sample consisted of 274 patients with a confirmed diagnosis of schizophrenia, and all of them completed the Simple Cope Style Questionnaire, Social Readjustment Rating Scale, and cognitive function assessment. A multivariate regression analysis was performed to investigate the possible correlations between cognitive function and stress, and the moderating effects of coping style on the relationship between stress and cognitive function were tested using the PROCESS macro for SPSS.

Results: Stress was negatively correlated with working memory. Negative coping but not positive coping moderated the relationship between stress and working memory in patients with schizophrenia, and the Johnson-Neyman technique showed that the moderating effect was significant only above this cutoff (38.32\% of all negative coping scores). This means that when exposed to similar stress, patients adopting high negative coping had worse working memory than those who did not.

Conclusion: These findings suggested that the assessment of stress and coping style may help estimate working memory impairment risk in patients with schizophrenia, and reducing negative coping may be a crucial intervention target to prevent further impairment of working memory in patients with schizophrenia suffering from great stress.

Keywords: cognitive function, life events, negative coping, positive coping

\section{Introduction}

As one of the most common symptoms in schizophrenia, cognitive impairment poses a risk to $>50 \%$ of patients with schizophrenia, especially in attention, memory, verbal fluency, and executive function. ${ }^{1}$ Previous research has suggested that cognitive impairment was consistently and strongly associated with functional outcome, ${ }^{2}$ living quality, ${ }^{3}$ and costs of care in individuals with schizophrenia; ${ }^{4}$ this indicated that it is crucial to represent risk factors for cognitive impairment in individuals with schizophrenia.

Stress is the nonspecific response of the body to stressors. ${ }^{5}$ Prior research has shown that the posttraumatic stress disorder (PTSD) group performed significantly 
worse than the non-PTSD group in the domains of attention, working memory, and executive function in patients with schizophrenia. ${ }^{6}$ Some studies have also proposed mechanisms by which stress-induced overexpression of immune factors causes destruction of integrity of the myelin sheath, leading to abnormal neuron circuit connections and information transmission. ${ }^{7,8}$ However, other studies showed that PTSD was not associated with increased cognitive impairment but was associated with more severe psychopathology. ${ }^{9}$ The divergence between the two findings may be associated with changes in positive and negative adaptive strategies in response to stress in individuals with schizophrenia. An important finding was that the emotion control could promote adaptive response to stress in schizophrenia. ${ }^{10}$

It has been proved that cognitive function is influenced by course of disease, positive and negative symptoms, and unhealthy behaviors such as smoking. ${ }^{11}$ However, previous studies have not determined the relationships between stress and domains of cognitive impairment in patients with schizophrenia. Therefore, it is essential to identify the relationship between stress and cognitive function, which can be targeted to assess risk factors for cognitive impairment and improve cognitive function in patients with schizophrenia.

As a potential risk factor, similar stress acts differently in individuals, ${ }^{12}$ suggesting the presence of other variables that moderate the relationship between stress and stress response. Coping style is a behavioral strategy adopted by individuals to cope with their own unstable state caused by stressors. ${ }^{13}$ The coping style has a moderating effect on the relationship between stress and stress response according to the stress and coping theory. ${ }^{14}$ Recent studies have shown that the maladaptive strategies were more strongly associated with psychopathology symptoms and stress response, while adaptive strategies showed no relationship with either psychopathology symptoms or stress response. ${ }^{15,16}$ Other studies have shown that less adaptive strategies were associated with worse psychopathology symptoms only at high levels of maladaptive strategies. ${ }^{17}$ These results may be due to the fact that stress-induced emotional cognitive regulation disorder leads to difficulties in the application of adaptive strategies. ${ }^{18}$ Moreover, the study also found that emotional management training could significantly improve emotional perception, cognitive function, and behavior in patients with schizophrenia. ${ }^{19}$ However, it is unknown whether the coping style might exert moderating effects on the relationship between stress and cognitive function in patients with schizophrenia. Therefore, investigating the effects of coping styles on cognitive function under stress is essential for improving cognitive function and alleviating psychopathology symptoms in patients with schizophrenia.

We hypothesized that an excessive stress would be related to worse cognitive function and that coping style had moderating effects on the relationship between stress and cognitive function in patients with schizophrenia. Specifically, the lowest level of cognitive impairment was observed in those who adopted more positive coping or less negative coping and had few stresses.

\section{Patients and methods Subjects}

A total of 274 inpatients with schizophrenia were recruited from the Ningxia Mental Health Center (Ningxia Ning-An Hospital). Inclusion criteria were as follows: patients of age 18-60 years old with confirmed diagnosis of schizophrenia according to the International Classification of Disease 10th revision. The exclusion criteria were inpatients with any mental retardation, organic central nervous system disorders, substance-related disorders but not including nicotine dependence, or severe somatic disorders.

\section{Measures}

\section{Cognitive function}

The Brief Assessment of Cognition in Schizophrenia (BACS) was designed to assess the most detrimental domains of cognition function, which were significantly correlated with outcome in patients with schizophrenia. ${ }^{20}$ Seven domains of cognition function including verbal memory, working memory, motor speed, verbal fluency, attention, executive function, and composite score are measured using the following seven tasks, respectively: Learning, Digital Sequencing, Token Motor, Category Instances, Controlled Oral Word Association, Symbol Coding, and Tower of London. Higher scores reflect better corresponding dimensions of cognitive function. The BACS composite scores were strongly related with composite scores of the standard battery for patients with schizophrenia $(r=0.76){ }^{21}$

\section{Coping style}

The Simple Coping Style Questionnaire (SCSQ) was used to evaluate the two scopes of coping style, including positive coping and negative coping. ${ }^{22}$ The ranking of SCSQ items by an interviewer range from 0 to 3 , in which, top marks of each domain indicate adopting more coping style of the domain. The positive coping domain consists of 12 items 
assessing positive coping characteristics derived from various activities, while the negative coping domain involves eight items assessing negative coping characteristics derived from various activities. Cronbach's alpha suggested strong consistent reliability, wherein the value was 0.89 for the positive coping domain and 0.78 for the negative coping domain. The confirmatory factor analysis suggested good structural validity. ${ }^{23}$

\section{Stress}

The Social Readjustment Rating Scale (SRRS) is applied to evaluate stress caused by life events over the past 12 months. ${ }^{24}$ This self-administered questionnaire consists of 43 items. The weight of these items ranges from 11 to 100 in terms of the severity of life events (eg, death of a spouse 100 , changing to a different line of work 36 , traffic violation 11). The level of stress is classified as being low $(\leq 149)$, mild (150-199), moderate (200-299), and major ( $\geq 300)$ according to the total scores of items. The research showed that those with low stress levels had a $30 \%$ or less risk for sick or mental disorders than the average population, and those with major stress levels had more possibilities to suffer from stress-related illness in the next year. ${ }^{25}$ A previous study supported the use of SRRS as a valid tool to measure stress-related outcomes. ${ }^{26}$

\section{Psychopathology syndrome}

The psychopathological symptoms are evaluated by psychiatrists using the Positive and Negative Syndrome Scale (PANSS) once a week after admission. The PANSS consists of three subscales representing positive, negative, and general psychiatric symptoms. The PANSS scores used in this study were derived from medical records at the second week after admission.

Demographic data (such as age, sex, and education), duration of disease, and PANSS scores were documented from medical records.

\section{Procedures}

The participants were inpatients who were admitted to the hospital recently. The patients were assessed by a psychiatrist in the second week after admission. If the patient's condition was stable, the questionnaire was administered and cognitive function assessment was performed.

The evaluation was conducted in a separate interview room. The evaluation order was as follows. First, respondents identified by psychiatrists signed the informed consent form before the questionnaire survey. Then, the respondents completed self-reporting questionnaires consisting of SCSQ and SRRS. Finally, the cognitive functions of the respondents were evaluated by the researchers, all of whom were certified psychological consultants and had received combined training including inclusion and exclusion criteria, evaluation order, and evaluation methods prior to the study.

The study was approved by the Ningxia Medical University Institutional Ethics Committee. All the participants provided written informed consent, and that the study was in accordance with the latest version of the Declaration of Helsinki.

\section{Data analysis}

First, descriptive statistical analysis was used to describe the characteristics of the sample; categorical variables were represented as percentage while quantitative variables were denoted as mean $\pm \mathrm{SD}$.

Second, multivariable linear regression analysis was used to investigate the possible association between the BACS domains (verbal memory, working memory, motor speed, attention, executive functions, verbal fluency, and composite score) and stress. The result of collinearity diagnostics showed that the variance inflation factor of all independent variables was between 1 and 2 . In this analysis, each domain in BACS was regarded as the dependent variable, while age, sex, course of disease, education, smoking, PANSS scores, and stress were regarded as the independent variables. The independent variable sex was assigned as "male $=1$, female $=2$ ". These two steps were performed using SPSS (IBM Corporation, version 20, 2011). ${ }^{44}$

Third, with the regression analysis results indicating that working memory was strongly related with stress in the second step (see Table 1), we further investigated moderation effects of coping style on stress and working memory in patients with schizophrenia. In the analysis, moderation was tested using the PROCESS macro for SPSS (IBM Corporation, version 20, 2011) with stress, positive coping, and negative coping centered, ${ }^{27}$ as well as the predictor of moderators such as positive coping and negative coping prior to the moderation analysis. Then, stress was inputted as the independent variable, working memory was inputted as the outcome variable, positive coping and negative coping were inputted as the moderator variables, and education, course of disease, and PANSS negative symptoms of schizophrenia were inputted as covariates, which were attributed 
to the fact that every variable of them was significantly correlated to working memory (see Table 1).

Parameters were set at $95 \%$ percentile-based confidence intervals (CIs).

Table I Multivariate regression analysis of the cognitive function with stress in patients with schizophrenia

\begin{tabular}{|c|c|c|c|}
\hline Variables & B & $P$-value & Model $P$-value \\
\hline Composite score & & & $\leq 0.001$ \\
\hline Age & 0.012 & 0.909 & \\
\hline Sex & 15.138 & 0.004 & \\
\hline Education, years & 6.287 & 0.000 & \\
\hline Course of disease, years & -1.308 & 0.000 & \\
\hline Smoking/no smoking & 4.061 & 0.487 & \\
\hline PANSS positive symptoms & 0.780 & 0.096 & \\
\hline PANSS negative symptoms & -0.479 & 0.214 & \\
\hline PANSS general symptoms & -0.209 & 0.523 & \\
\hline Stress & -0.008 & 0.424 & \\
\hline \multicolumn{4}{|l|}{ Verbal memory } \\
\hline Age & -0.002 & 0.791 & \\
\hline Sex & 0.583 & 0.192 & \\
\hline Education, years & 0.302 & 0.000 & \\
\hline Course of disease, years & -0.106 & 0.000 & $\leq 0.00 \mathrm{I}$ \\
\hline Smoking/no smoking & 0.237 & 0.636 & \\
\hline PANSS positive symptoms & 0.007 & 0.861 & \\
\hline PANSS negative symptoms & 0.012 & 0.710 & \\
\hline PANSS general symptoms & -0.006 & 0.842 & \\
\hline Stress & 0.000 & 0.733 & \\
\hline \multicolumn{4}{|l|}{ Working memory } \\
\hline Age & 0.007 & 0.624 & \\
\hline Sex & -0.377 & 0.601 & \\
\hline Education, years & 0.745 & 0.000 & \\
\hline Course of disease, years & -0.083 & 0.041 & $\leq \mathbf{0 . 0 0 I}$ \\
\hline Smoking/no smoking & 0.347 & 0.667 & \\
\hline PANSS positive symptoms & 0.103 & 0.111 & \\
\hline PANSS negative symptoms & -0.116 & 0.030 & \\
\hline PANSS general symptoms & -0.013 & 0.773 & \\
\hline Stress & -0.003 & 0.040 & \\
\hline \multicolumn{4}{|l|}{ Motor speed } \\
\hline Age & -0.002 & 0.955 & \\
\hline Sex & 6.672 & 0.001 & \\
\hline Education, years & 0.651 & 0.035 & \\
\hline Course of disease, years & -0.404 & 0.001 & \\
\hline Smoking/no smoking & -0.212 & 0.927 & $\leq \mathbf{0 . 0 0 I}$ \\
\hline PANSS positive symptoms & 0.103 & 0.577 & \\
\hline PANSS negative symptoms & -0.032 & 0.834 & \\
\hline PANSS general symptoms & -0.061 & 0.635 & \\
\hline Stress & -0.007 & 0.071 & \\
\hline \multicolumn{4}{|l|}{ Attention } \\
\hline Age & -0.021 & 0.478 & \\
\hline Sex & 2.776 & 0.058 & \\
\hline Education, years & 1.922 & 0.000 & \\
\hline Course of disease, years & -0.386 & 0.000 & $\leq \mathbf{0 . 0 0 I}$ \\
\hline Smoking/no smoking & 1.854 & 0.257 & \\
\hline PANSS positive symptoms & 0.188 & 0.151 & \\
\hline PANSS negative symptoms & -0.08 I & 0.454 & \\
\hline PANSS general symptoms & -0.007 & 0.942 & \\
\hline Stress & -0.002 & 0.402 & \\
\hline
\end{tabular}

(Continued)
Table I (Continued)

\begin{tabular}{l|l|l|l}
\hline Variables & B & $P$-value & Model $\boldsymbol{P}$-value \\
\hline Executive function & & & \\
Age & -0.004 & 0.732 & \\
Sex & -1.250 & $\mathbf{0 . 0 3 4}$ & \\
Education, years & 0.502 & $\mathbf{0 . 0 0 0}$ & \\
Course of disease, years & -0.058 & 0.082 & $\leq \mathbf{0 . 0 0 I}$ \\
Smoking/no smoking & $-0.21 \mathrm{I}$ & 0.748 & \\
PANSS positive symptoms & -0.046 & 0.377 & \\
PANSS negative symptoms & -0.070 & 0.108 & \\
PANSS general symptoms & 0.048 & 0.195 & \\
Stress & -0.001 & 0.314 & \\
Verbal fluency & & & \\
Age & 0.034 & 0.493 & \\
Sex & 6.733 & $\mathbf{0 . 0 0 7}$ & \\
Education, years & 2.165 & $\mathbf{0 . 0 0 0}$ & \\
Course of disease, years & -0.270 & 0.056 & $\leq \mathbf{0 . 0 0 I}$ \\
Smoking/no smoking & 2.047 & 0.464 & \\
PANSS positive symptoms & 0.427 & 0.057 & \\
PANSS negative symptoms & -0.193 & 0.294 & \\
PANSS general symptoms & -0.170 & 0.277 & \\
Stress & 0.006 & 0.229 & \\
\hline
\end{tabular}

Note: Bold represents significant $P$-values.

\section{Results}

\section{The participants' characteristics}

A total of 274 patients with schizophrenia including 134 males $(48.9 \%)$ and 140 females $(51.1 \%)$ were included in the study with a mean age of 33.93 years $(S D=20.56)$, an average course of disease of 9.32 years $(\mathrm{SD}=7.25)$, and a mean level of education of 11.17 years $(\mathrm{SD}=2.78)$; most of the participants were nonsmokers $(75.2 \%)$. The average score of stress was 264.92 (SD =209.393), showing a moderate severity of stress according to scores on the SRRS. ${ }^{28}$ Mean and SDs of PANSS subscales, cognitive function domains, and cope style scores of participants are shown in Table 2.

\section{The correlation between stress and cognitive function}

Stress was significantly related to working memory, and significant correlations were found between sex and motor speed, verbal fluency, executive function and composite score in patients with schizophrenia.

However, there were no significant correlations among verbal memory, motor speed, attention, executive function, verbal fluency, composite score, and stress. In addition, the result showed correlation among course of disease, education, negative symptoms, and working memory in individuals with schizophrenia (see Table 1). 
Table 2 Demographic and assessment results of sample characteristics in patients with schizophrenia $(n=274)$

\begin{tabular}{|c|c|}
\hline Variables & Mean \pm SD or $n(\%)$ \\
\hline Age, years & $33.93 \pm 20.56$ \\
\hline \multicolumn{2}{|l|}{ Sex } \\
\hline Male & I 34 (48.9\%) \\
\hline Female & 140 (5I. I\%) \\
\hline Education, years & $11.17 \pm 2.78$ \\
\hline \multicolumn{2}{|l|}{ Smoking status } \\
\hline Smoking & $68(24.8 \%)$ \\
\hline No smoking & $206(75.2 \%)$ \\
\hline Course of disease & $9.32 \pm 7.25$ \\
\hline \multicolumn{2}{|l|}{ PANSS symptoms } \\
\hline PANSS positive symptoms & $18.97 \pm 6.04$ \\
\hline PANSS negative symptoms & $|8.8| \pm 6.57$ \\
\hline PANSS general symptoms & $32.90 \pm 9.92$ \\
\hline Stress & $264.92 \pm 209.39$ \\
\hline \multicolumn{2}{|l|}{ Cognitive function } \\
\hline Verbal memory & $10.57 \pm 3.09$ \\
\hline Working memory & $17.15 \pm 5.24$ \\
\hline Motor speed & $65.36 \pm 14.11$ \\
\hline Verbal fluency & $58.93 \pm 17.80$ \\
\hline Attention & $36.66 \pm 11.37$ \\
\hline Executive function & $11.23 \pm 4.11$ \\
\hline Composite score & $199.89 \pm 40.26$ \\
\hline \multicolumn{2}{|l|}{ Coping style } \\
\hline Positive coping & $10.49 \pm 5.59$ \\
\hline Negative coping & $19.50 \pm 6.82$ \\
\hline
\end{tabular}

Abbreviations: PANSS, Positive and Negative Syndrome Scale, SD, standard deviation.

\section{The moderating effects of coping style on the relationship between stress and working memory}

Outcome of the PROCESS macro for SPSS indicated that the assumption was partly justified for moderating effects of coping style. The model of positive coping as a moderator variable was significant, $R^{2}=0.21, F(6,267)=12.13$,
$P<0.001$. However, positive coping did not moderate the relationship between stress and working memory in the sample (see Table 3); furthermore, the interaction term did not strongly improve forecasting power of the model, $\Delta R^{2}=0.00$, $F(1,267)=0.05, P=0.772$ (see Figure 1 and Table 3).

The model of negative coping as a moderator variable was significant, $R^{2}=0.23, F(6,267)=13.55, P<0.001$, and the interaction term obviously improved forecasting power of the model, $\Delta R^{2}=0.013, F(1,267)=5.16, P=0.035$, indicating that negative coping moderated the relationship between stress and working memory (see Figure 2 and Table 3). The Johnson-Neyman technique showed that the moderating effect was not significant when the negative coping score was $<2.06$ (equivalent to 21.56 in noncentered scores, and $61.68 \%$ of all negative coping scores were below the cutoff), but significant above this cutoff (38.32\% of scores); this indicated that negative coping moderated the relationship between stress and working memory in patients with schizophrenia when negative coping was high.

\section{Discussion}

To determine the association between stress and different dimensions of cognitive function, the study focused on whether coping style influences the effect of stress on cognitive function in patients with schizophrenia. The results indicated that stress was related to working memory; this finding was consistent with that of the previous study. Another study indicated that excessive dopamine activity in the prefrontal cortex under stress had a negative impact on working memory in humans. ${ }^{29}$ In addition, this study also showed that negative coping moderated the relationship between stress and working memory, while positive coping did not. Specifically, individuals adopting high negative

Table 3 Model results predicting moderation of coping style in the relationship between stress and working memory

\begin{tabular}{|c|c|c|c|c|c|c|c|}
\hline Analysis & Predictor & $\begin{array}{l}\text { Regression } \\
\text { coefficient }\end{array}$ & $\begin{array}{l}\text { Standard } \\
\text { error }\end{array}$ & $t$ & $P$-value & $\begin{array}{l}\text { Lower limit of } \\
95 \% \mathrm{Cl}\end{array}$ & $\begin{array}{l}\text { Upper limit of } \\
95 \% \mathrm{Cl}\end{array}$ \\
\hline \multirow[t]{6}{*}{ Model I } & Education & 0.391 & 0.104 & 7.069 & 0.000 & 0.530 & 0.940 \\
\hline & Course of disease & -0.107 & 0.039 & -1.970 & 0.050 & -0.155 & 0.000 \\
\hline & Negative symptoms & -0.131 & 0.044 & -2.370 & 0.018 & -0.191 & -0.018 \\
\hline & Stress & -0.111 & 0.001 & -1.959 & 0.051 & -0.006 & 0.000 \\
\hline & Positive coping & -0.010 & 0.053 & -0.177 & 0.860 & -0.113 & 0.094 \\
\hline & Interaction term & 0.016 & 0.000 & 0.291 & 0.772 & 0.000 & 0.001 \\
\hline \multirow[t]{6}{*}{ Model 2} & Education & 0.390 & 0.110 & 6.700 & 0.000 & 0.519 & 0.951 \\
\hline & Course of disease & -0.100 & 0.039 & $-|.86|$ & 0.064 & -0.149 & 0.004 \\
\hline & Negative symptoms & -0.120 & 0.044 & -2.175 & 0.031 & -0.182 & -0.009 \\
\hline & Stress & -0.072 & 0.001 & -1.215 & 0.225 & -0.005 & 0.001 \\
\hline & Negative coping & 0.013 & 0.046 & 0.226 & 0.821 & -0.079 & 0.100 \\
\hline & Interaction term & -0.122 & 0.000 & -2.116 & 0.035 & -0.001 & 0.000 \\
\hline
\end{tabular}

Notes: Stress measured by the Social Readjustment Rating Scale; positive coping and negative coping measured by the Simple Cope Style Questionnaire (SCSQ). Interaction term is the multiplied effects of stress and positive coping (Model I) and stress and negative coping (Model 2). 


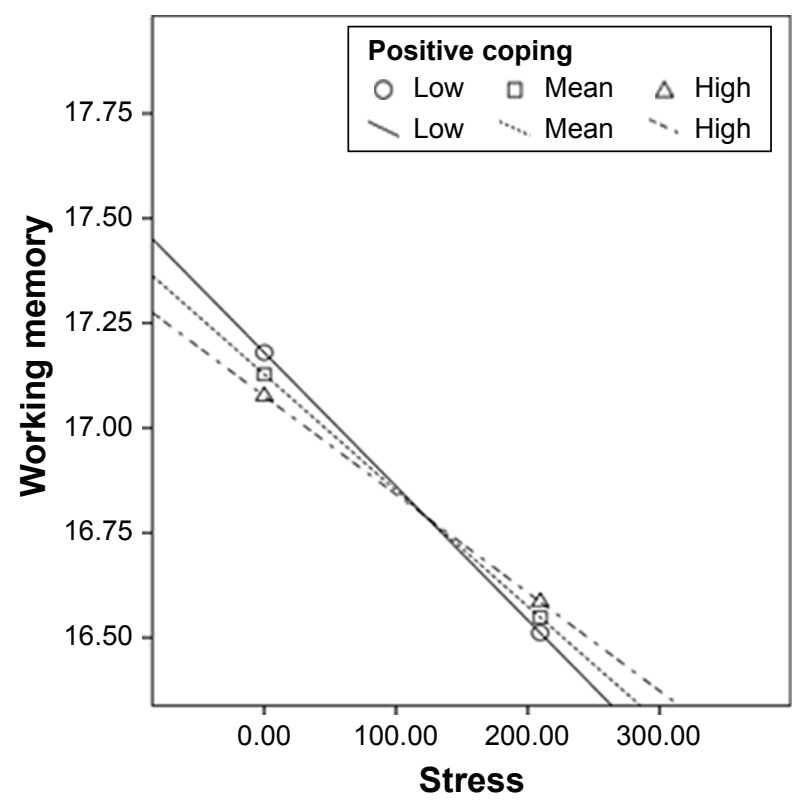

Figure I Effects of stress on working memory plotted by low, mean, and high positive coping.

Note: Low, mean, and high positive coping were mean - SD, mean, and mean + SD of positive coping scores.

coping demonstrated worse working memory when suffering from similar stress. These findings were limited only to negative coping, which was attributed to the fact that the interaction of positive coping and stress was not significantly related to working memory. This was partly in line with our original assumption, thus improving the credibility of the

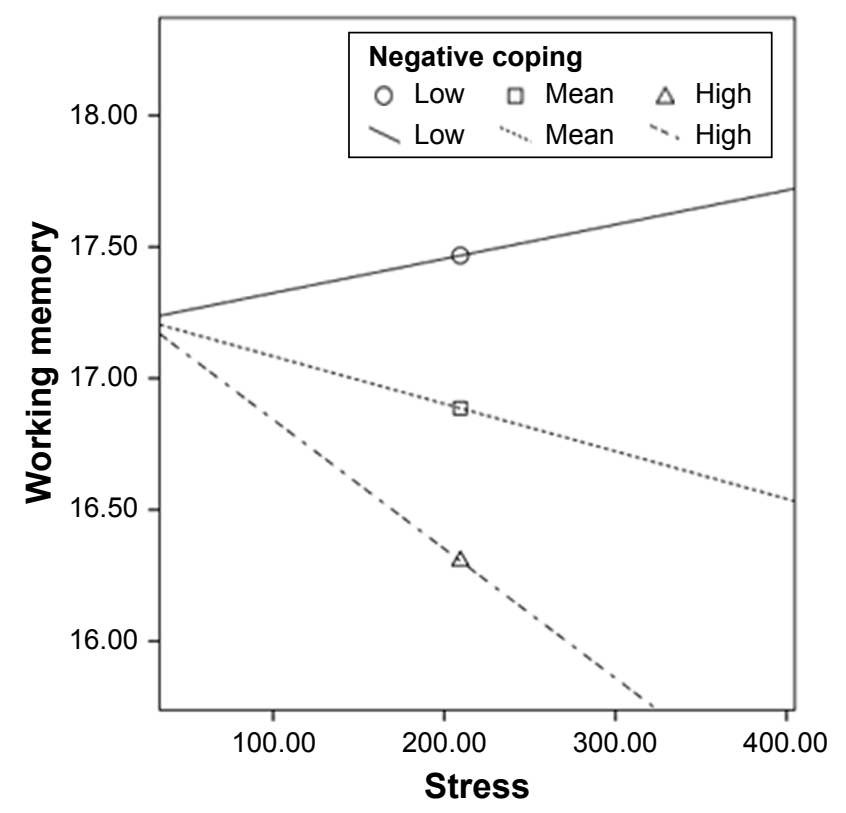

Figure 2 Effects of stress on working memory plotted by low, mean, and high negative coping.

Note: Low, average, and high negative coping were mean -SD, mean, and mean + SD of negative coping scores. rationale of the stress and coping theory. ${ }^{30}$ However, it may not be the appropriate model from which to assess working memory impairment in those adopting more positive coping. This finding also suggests that negative coping may be a crucial intervention target to prevent further impairment of working memory for individuals with schizophrenia exposed to high stress.

Some studies on stress and cognitive impairment may help explain this phenomenon. Stress may lead to maladaptive responses that could elevate cortisol levels, ${ }^{31,32}$ which were significantly related to impaired memory function in schizophrenia. ${ }^{33}$ The maladaptive emotion regulation significantly predicted an increased affective and reduced cortisol response. ${ }^{34}$ In addition, stress was strongly associated with excessive expression of inflammatory factors such as tumor necrosis factor $\alpha$, IL-8, and IL-10, which caused destruction of myelin sheath integrity and contributed to cognitive deficits. ${ }^{35,36}$ The cognitive deficits were strongly associated with the ineffective use of adaptive strategies, and the remediation of cognitive deficits contributing to ineffective adaptive strategies may facilitate reduced mood symptomatology. ${ }^{37}$ Therefore, maladaptive emotion regulation strategies and cognitive function remediation may be essential to prevent further deterioration of symptoms and mood disorders for schizophrenia patients with more stress.

This study filled in the gaps of previous studies, in which clinical factors have influenced cognitive function in patients with schizophrenia, ${ }^{38,39}$ owing to the identification of relationships between stress and cognitive function in patients with schizophrenia. More importantly, we identified a significant interaction in which negative coping moderated the relationship between stress and working memory, which may be crucial in assessing working memory impairment risk and determining intervention targets. Furthermore, it may be beneficial to conduct working memory training programs with behavioral interventions to prevent further impairment of working memory in patients with schizophrenia exposed to more stress. These behavioral interventions might be effective especially in reducing negative coping in patients with schizophrenia exposed to serious stress, such as anxiety management, ${ }^{40}$ cognitive adaptation training, ${ }^{41}$ and Awareness and Coping with Emotion in Schizophrenia intervention. ${ }^{42}$

The present study has several limitations. It is impossible to elaborate any causal relationship through a cross-sectional study. The inpatients may have more serious symptoms than outpatients, which may introduce bias. However, quite a large sample size with sex distribution balanced, comparatively, makes it more possible for generalizability. The application 
of self-report measures in studies makes it impossible for us to exclude the likelihood of symptoms bias in responding. These limitations may be reduced in the future through longitudinal studies by collecting more samples of outpatients and adopting multiple methods to evaluate series of variables to decrease the possibility of bias. Owing to the fact that psychotropic drug intake in the year prior to admission cannot be a precise measure for the difference of medication adherence in individuals with schizophrenia, the psychotropic drugs were not included in the study. The inclusion criteria should include medication adherence in the future, which will help to accurately measure psychotropic drug intake of outpatients. In addition, the early life stress events were not restrained in the study because the brain nerve is malleable under the influence of behavior and environment over a long period, ${ }^{43}$ and because of the unclear relationship between early life events and cognitive function in the previous studies. It may be a good option to design controlled study for investigating the effect of early life events on cognitive function of patients with schizophrenia in the future.

\section{Conclusion}

These results showed that stress was related to working memory in one of the cognitive function domains. Negative coping moderated the relationship between stress and working memory only under high negative coping, while positive coping did not have the moderating effect. More specifically, more stress was associated with worse working memory only in those adopting high negative coping. The outcome of this study demonstrates that the evaluation for stress and coping styles might be necessary when assessing cognitive function impairment risk in patients with schizophrenia. In addition, it may be beneficial to adopt collaboration interventions to prevent further impairment of working memory in patients with schizophrenia exposed to high stress.

\section{Acknowledgment}

The work was greatly supported by the National Natural Sciences Foundation of China (NSFC; grant no 81460214).

\section{Author contributions}

Professor KW was in charge of study design, monitored the study, and revised it critically for important intellectual content in the paper. Professor JL was accountable for all aspects of the work in ensuring that questions related to the work are appropriately resolved. Dr XZ designed the study, undertook the statistical analysis, and finished the first draft of the manuscript. XX wrote the study protocol. CX, JZ, $\mathrm{XZ}$, and LM collected the data and carried on interpretation of data. All authors contributed to data analysis, drafting and revising the article, gave final approval of the version to be published, and agree to be accountable for all aspects of the work.

\section{Disclosure}

The authors report no conflicts of interest in this work.

\section{References}

1. Moustafa AA, Garami JK, Mahlberg J, et al. Cognitive function in schizophrenia: conflicting findings and future directions. Rev Neurosci. 2016;27(4):435-448.

2. Wojtalik JA, Smith MJ, Keshavan MS, Eack SM. A systematic and meta-analytic review of neural correlates of functional outcome in schizophrenia. Schizophr Bull. 2017;43(6):1329-1347.

3. Bambini V, Arcara G, Bechi M, Buonocore M, Cavallaro R, Bosia M. The communicative impairment as a core feature of schizophrenia: frequency of pragmatic deficit, cognitive substrates, and relation with quality of life. Compr Psychiatry. 2016;71:106-120.

4. Reeder C, Harris V, Pickles A, Patel A, Cella M, Wykes T. Does change in cognitive function predict change in costs of care for people with a schizophrenia diagnosis following cognitive remediation therapy? Schizophr Bull. 2014;40(6):1472-1481.

5. Selye H. The stress concept. Can Med Assoc J. 1976;115(8):718.

6. Fan X, Henderson DC, Nguyen DD, et al. Posttraumatic stress disorder, cognitive function and quality of life in patients with schizophrenia. Psychiatry Res. 2008;159(1-2):140-146.

7. Small SA. Isolating pathogenic mechanisms embedded within the hippocampal circuit through regional vulnerability. Neuron. 2014;84(1): 32-39.

8. Deak T, Kudinova A, Lovelock DF, Gibb BE, Hennessy MB. A multispecies approach for understanding neuroimmune mechanisms of stress. Dialogues Clin Neurosci. 2017;19(1):37-53.

9. Peleikis DE, Varga M, Sundet K, Lorentzen S, Agartz I, Andreassen OA. Schizophrenia patients with and without post-traumatic stress disorder (PTSD) have different mood symptom levels but same cognitive functioning. Acta Psychiatr Scand. 2013;127(6):455-463.

10. Tully LM, Lincoln SH, Hooker CI. Lateral prefrontal cortex activity during cognitive control of emotion predicts response to social stress in schizophrenia. Neuroimage Clin. 2014;6(6):43-53.

11. Kishi T, Moriwaki M, Kawashima K, et al. Investigation of clinical factors influencing cognitive function in Japanese schizophrenia. Neurosci Res. 2010;66(4):340-344.

12. Selye H. Forty years of stress research: principal remaining problems and misconceptions. Can Med Assoc J. 1976;115(1):53-56.

13. Lazarus RS. The psychology of stress and coping. Issues Ment Health Nurs.1985;7(1-4):399-418.

14. Lazarus RS. Coping theory and research: past, present, and future. Psychosom Med. 1993;55(3):234-247.

15. Krkovic K, Clamor A, Lincoln TM. Emotion regulation as a predictor of the endocrine, autonomic, affective, and symptomatic stress response and recovery. Psychoneuroendocrinology. 2018;94:112-120.

16. Aldao A, Nolen-Hoeksema S. Specificity of cognitive emotion regulation strategies: a transdiagnostic examination. Behav Res Ther. 2010; 48(10):974-983.

17. Aldao A, Nolen-Hoeksema $S$. When are adaptive strategies most predictive of psychopathology? J Abnorm Psychol. 2012;121(1):276-281.

18. Raio CM, Orederu TA, Palazzolo L, Shurick AA, Phelps EA. Cognitive emotion regulation fails the stress test. Proc Natl Acad Sci US A. 2013;110(37):15139-15144. 
19. Hodel B, Brenner HD. [A training program for coping with maladaptive emotions of schizophrenic patients. Initial results and experiences]. Nervenarzt. 1996;67(7):564-571.

20. Keefe RS, Poe M, Walker TM, Harvey PD. The relationship of the Brief Assessment of Cognition in Schizophrenia (BACS) to functional capacity and real-world functional outcome. J Clin Exp Neuropsychol. 2006;28(2):260-269.

21. Keefe RS, Goldberg TE, Harvey PD, Gold JM, Poe MP, Coughenour L. The Brief Assessment of Cognition in Schizophrenia: reliability, sensitivity, and comparison with a standard neurocognitive battery. Schizophr Res. 2004;68(2-3):283-297.

22. Du B, Ma X, Ou X, Jin Y, Ren P, Li J. The prevalence of posttraumatic stress in adolescents eight years after the Wenchuan earthquake. Psychiatry Res. 2018;262:262-269.

23. Hai YN. A preliminary study on reliability and validity of simple cope style questionnaire. Chin J Clin Psychol. 1998;6(2):114-115.

24. Holmes TH, Rahe RH. The Social Readjustment Rating Scale. J Psychosom Res. 1967;11(2):213-218.

25. Smith CK, Cullison SW, Polis E, Holmes TH. Life change and illness onset: importance of concepts for family physicians. J Fam Pract. 1978; 7(5):975-981.

26. Jiang Y, Yang M, Lv Q, et al. Prevalence of psychological disorders, sleep disturbance and stressful life events and their relationships with disease parameters in Chinese patients with ankylosing spondylitis. Clin Rheumatol. 2018;37(2):407-414.

27. Hayes AF. Introduction to Mediation, Moderation, and Conditional Process Analysis: A Regression-Based Approach. New York: Guilford Press; 2013.

28. Bieliauskas LA, Strugar DA. Sample size characteristics and scores on the Social Readjustment Rating Scale. J Psychosom Res. 1976;20(3): 201-205.

29. Bahari Z, Meftahi GH, Meftahi MA. Dopamine effects on stress-induced working memory deficits. Behav Pharmacol. 2018;29(7):584-591.

30. Lazarus RS. Psychological stress and coping in adaptation and illness. Int J Psychiatry Med. 1974;5(4):321-333.

31. Shekhar A, Truitt W, Rainnie D, Sajdyk T. Role of stress, corticotrophin releasing factor (CRF) and amygdala plasticity in chronic anxiety. Stress. 2005;8(4):209-219.

32. Tull MT, Berghoff CR, Wheeless LE, Cohen RT, Gratz KL. PTSD symptom severity and emotion regulation strategy use during trauma cue exposure among patients with substance use disorders: associations with negative affect, craving, and cortisol reactivity. Behavior Ther. 2018;49(1):57-70.
33. Havelka D, Prikrylova-Kucerova H, Prikryl R, Ceskova E. Cognitive impairment and cortisol levels in first-episode schizophrenia patients. Stress. 2016;19(4):383-389.

34. Krkovic K, Clamor A, Lincoln TM. Emotion regulation as a predictor of the endocrine, autonomic, affective, and symptomatic stress response and recovery. Psychoneuroendocrinology. 2018;94:112-120.

35. Wolkow A, Aisbett B, Ferguson SA, Reynolds J, Main LC. Psychophysiological relationships between a multi-component self-report measure of mood, stress and behavioural signs and symptoms, and physiological stress responses during a simulated firefighting deployment. Int J Psychophysiol. 2016;110:109-118.

36. Deak T, Kudinova A, Lovelock DF, Gibb BE, Hennessy MB. A multispecies approach for understanding neuroimmune mechanisms of stress. Dialogues Clin Neurosci. 2017;19(1):37-53.

37. Rowland JE, Hamilton MK, Lino BJ, et al. Cognitive regulation of negative affect in schizophrenia and bipolar disorder. Psychiatry Res. 2013;208(1):21-28.

38. Alloza C, Bastin ME, Cox SR, et al. Central and non-central networks, cognition, clinical symptoms, and polygenic risk scores in schizophrenia. Hum Brain Mapp. 2017;38(12):5919-5930.

39. Krkovic K, Moritz S, Lincoln TM. Neurocognitive deficits or stress overload: why do individuals with schizophrenia show poor performance in neurocognitive tests? Schizophr Res. 2017;183:151-156.

40. Buonocore M, Bosia M, Bechi M, et al. Targeting anxiety to improve quality of life in patients with schizophrenia. Eur Psychiatry. 2017;45:129-135.

41. Fredrick MM, Mintz J, Roberts DL, et al. Is cognitive adaptation training (CAT) compensatory, restorative, or both? Schizophr Res. 2015; 166(1-3):290-296.

42. Caponigro JM, Moran EK, Kring AM, Moskowitz JT. Awareness and coping with emotion in schizophrenia: acceptability, feasibility and case illustrations. Clin Psychol Psychother. 2014;21(4):371-380.

43. Aumann TD. Environment- and activity-dependent dopamine neurotransmitter plasticity in the adult substantia nigra. J Chem Neuroanat. 2016;73:21-32.

44. IBM Corporation. IBM SPSS Statistics for Windows. Version 20.0. Armonk, New York: IBM Corporation; 2011.
Neuropsychiatric Disease and Treatment

\section{Publish your work in this journal}

Neuropsychiatric Disease and Treatment is an international, peerreviewed journal of clinical therapeutics and pharmacology focusing on concise rapid reporting of clinical or pre-clinical studies on a range of neuropsychiatric and neurological disorders. This journal is indexed on PubMed Central, the 'PsycINFO' database and CAS,
Dovepress

and is the official journal of The International Neuropsychiatric Association (INA). The manuscript management system is completely online and includes a very quick and fair peer-review system, which is all easy to use. Visit http://www.dovepress.com/testimonials.php to read real quotes from published authors. 